\title{
Adhesión de fragmentos dentales con collage
}

\section{Dental fragments collage adhesion}

\author{
Morales, Byron ${ }^{1}$, Pinos, Patricia ${ }^{1}$ y Cordero, Augusta $^{1}$ \\ ${ }^{1}$ Universidad Católica de Cuenca. \\ Cuenca 010574, Ecuador. \\ *byronmorales@yahoo.com
}

\begin{abstract}
Resumen
Este artículo describe el caso clínico de un paciente de 19 años de edad, que recibió un golpe que produjo la fractura coronaria horizontal del incisivo central superior con exposición del paquete vásculo nervioso. La fractura involucró lesiones en los tejidos duros del diente, sin afectar el surco gingival y tejidos blandos. Dentro del plan de tratamiento se estableció realizar tratamiento endodóntico de la pieza 1.1, cementación de un poste de fibra de vidrio, y la unión de este y el fragmento dental mediante un sistema adhesivo y el uso de una resina compuesta, con el fin de preservar la pieza dental manteniendo su salud periodontal, estética y funcionalidad. En el control a los seis meses después de realizado el tratamiento se pudo observar que existía estabilidad de color, buena salud periodontal además de función masticatoria adecuada.
\end{abstract}

Palabras clave: fractura dental, adhesión, fragmentos dentales.

\begin{abstract}
This article describes the clinical case of a 19-year-old patient who received a fracture of the upper central incisor with exposure of the nervous vasopressor pack, and a complicated horizontal coronary fracture, due to the fact that it is a fracture of the amelodentin with pulp involvement. The fracture involved lesions on the hard tissues of the tooth, without affecting the gingival sulcus and soft tissues. Within the treatment plan was established to perform endodontic treatment of piece 1.1, cementation of a fiberglass post, and the union of this and the dental fragment through an adhesive system and the use of a composite resin, in order to preserve The dental piece maintaining its periodontal health, esthetics and functionality. At the control six months after the treatment, it was observed that there was color stability, good periodontal health and adequate masticatory function.
\end{abstract}

Key words: dental fracture, adhesion, dental fragments.

\section{Introducción}

Dentro de todas las especialidades, la odontología restauradora tiene como función tratar diversas lesiones dentales, con diferentes manifestaciones, por lo cual puede ser necesario la reposición artificial de los tejidos afectados. Las restauraciones dentales tienen como misión devolver anatomía, funcionalidad, estética tanto en el sector anterior como en el posterior, considerando los aspectos y necesidades que cada sector requiere.

La fractura dental coronaria es la lesión traumática más frecuente que se presenta en los dientes permanentes. ${ }^{1}$ Las piezas más comúnmente afectadas por traumatismos son los incisivos superiores, con un reporte del $96 \%$ de todas las fracturas coronarias ( $80 \%$ incisivos centrales y $16 \%$ incisivos laterales) $)^{2}$

De manera general los profesionales de la Odontología, manejan lesiones generadas ya sea por caries, o por traumatismos, producto de accidentes o actividades extremas. Los cuales pueden ser de diferentes tipos, demandando de parte del profesional habilidades, destrezas y conoci- mientos necesarios para resolver el caso de la manera más adecuada. Es importante mencionar que dependiendo del traumatismo y afección de los tejidos se requiera involucrar a especialistas de otras áreas (Endodoncia, Periodoncia, Cirugía). Gracias a los avances que ha presentado la odontología restauradora y adhesiva, la adhesión es el factor fundamental para lograr este tipo de restauraciones.

Es importante tener en cuenta que existe una clasificación de las fracturas dentales, las mismas que son de gran importancia para determinar un tratamiento y un pronóstico adecuado. La Clasificación de las lesiones según la OMS, Louis H. Berman menciona que las fracturas coronarias pueden ser: a) Fisuras y fracturas del esmalte b) Fractura amelodentinaria sin afectación pulpar (Fractura coronaria no complicada) c) Fractura amelodentinaria con afectación pulpar (Fractura coronaria complicada).

En la actualidad no contamos con ningún material restaurador que pueda igualar a los tejidos naturales, en relación a su resistencia y estética, con el transcurso del tiempo hemos podido ser partícipes del avance tecnológico para 
lograr resultados muy conservadores. Uno de los primeros trabajos registrados es el de Chosack y Eidelman publicado en 1964, quienes usaron grabado en el esmalte y resina compuesta para adherir un segmento fracturado al remanente. A su vez, Spasser en 1977 realizó el collage de un diente con una fractura que afecto a la cámara pulpar. ${ }^{3}$

Por su parte Baratieri en 1989 reportó el caso de una fractura coronaria intra-ósea con invasión del grosor biológico, este caso tuvo un seguimiento de seis años con excelente estado de salud periodontal y buena estética. ${ }^{3}$

Calixto y colaboradores en 1999 describieron un caso de fractura amplia en un incisivo central superior, el cual recibió pulpectomia a consecuencia de una fractura. Se realizó blanqueamiento dental y luego de 5 días el collage del fragmento dental, previa inserción de un poste radicular estético para mejorar la retención de la restauración.

Busato en el 2002 señala que aunque la preparación del bisel es importante para mejorar la retención, es preferible no realizarlo pues se exponen una mayor cantidad de material restaurador en la superficie, limitando su uso a discrepancias estéticas en el post operatorio.

Los traumatismos bucodentales son accidentes frecuentes que afectan negativamente al individuo en su proyección psicológica y social. Deben ser considerados como una situación de urgencia estomatológica, que requiere de diagnóstico certero y tratamiento oportuno por parte del estomatólogo general integral. ${ }^{4}$

Se indica que el éxito del tratamiento, usando la técnica de Collage consiste en mantener hidratado el fragmento dental en un PH adecuado colocando el mismo en leche o en la boca del paciente y la asistencia inmediata del paciente después de la fractura dentaria 8 y que el fracaso producido al adherir el fragmento dental al diente es porque no se utiliza los materiales recomendados y se presenta una falta de estética, fonética y funcionalidad. ${ }^{5}$

Es importante tener en cuenta que existen varias alternativas de tratamiento, y para lograr el éxito es necesario realizar un diagnóstico individual considerando diversos factores como son: la edad del paciente, la extensión y grado de fractura coronaria, la presencia o no de compromiso pulpar, el grado de desarrollo radicular, tipo de dentición en la que se encuentra el paciente, las características oclusales, la afección de estructuras adyacentes y el tiempo transcurrido luego del traumatismo. ${ }^{6}$

\section{Presentación del Caso Clínico}

Paciente de sexo masculino de 19 años de edad que acude a la consulta debido a una fractura en el incisivo central superior derecho, en la anamnesis el paciente relata que recibió un golpe de puño por causa de una pelea, produciéndose el desprendimiento de una parte del diente ya que sintió en su boca el fragmento dental, el paciente asiste de inmediato para revisión profesional, pero no lleva consigo el fragmento dental, el mismo que él comenta que ha mantenido en agua común hasta el momento, se le sugiere de inmediato traer el remanente dental para usarlo dentro del plan de tratamiento.

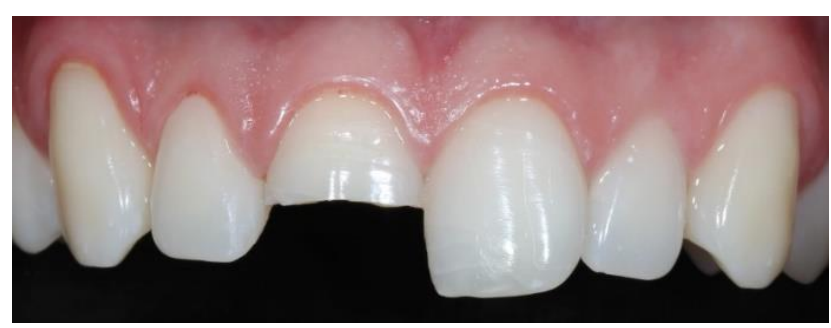

Fig. 1. Fractura del Incisivo Central Derecho

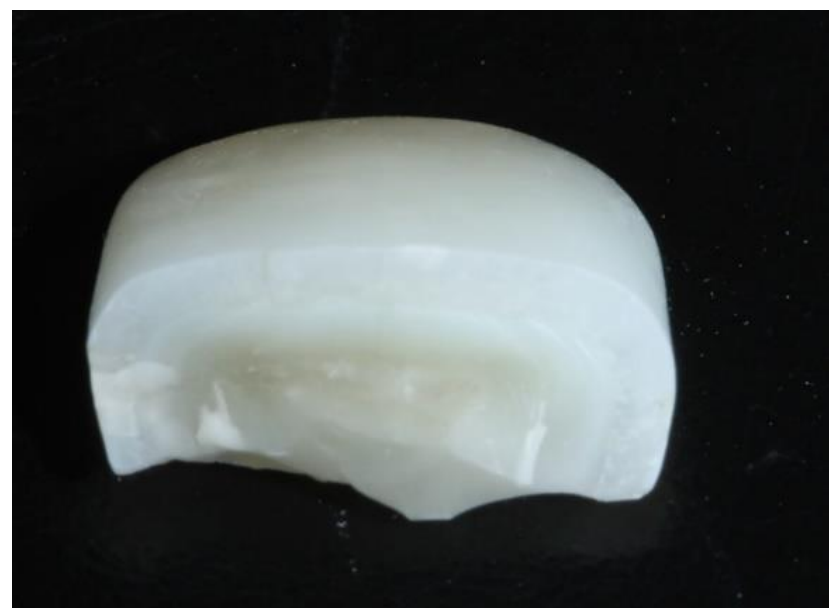

Fig. 2. Fragmento Dentario

En la inspección intraoral se observa ausencia parcial de la corona anatómica de la pieza número 11 , observándose una fractura amelodentinaria horizontal coronaria complicada con afección pulpar, pero que no involucra el tercio gingival. En la radiografía periapical se pudo distinguir la fractura, (horizontal) y la integridad tanto radicular como ósea, además de indicios de daño en las piezas vecinas. Una vez realizados todos los elementos de diagnóstico como son Historia clínica, radiografías y fotografías, se le explica la situación al paciente y se elabora la propuesta del plan de tratamiento. El paciente luego de analizar el plan de tratamiento pregunta "¿Cómo me va a quedar la restauración y que tipos de materiales se van a usar en la reconstrucción?", también indica que la fractura del diente le está causando un daño psicológico porque no quiere asistir a la universidad ya que no puede hablar ni sonreír. Estos factores fueron tomados en cuenta para poder realizar la restauración con la mayor celeridad y tratar de cubrir las expectativas del paciente tanto estéticas, funcionales y psicológicas.

Se procedió a anestesiar al paciente y a realizar el tratamiento de conducto de la pieza afectada, el fragmento coronal fue guardado por el paciente en un frasco en agua común, que se asemeja a los estudios realizado por Chosak 
y Eidelman en 1964 y los de Baratieri en 1998, en donde mencionan que mantienen los fragmentos limpios en un contenedor con agua estéril en refrigeración para asegurar la hidratación y por ende la estabilidad de color de los fragmentos.

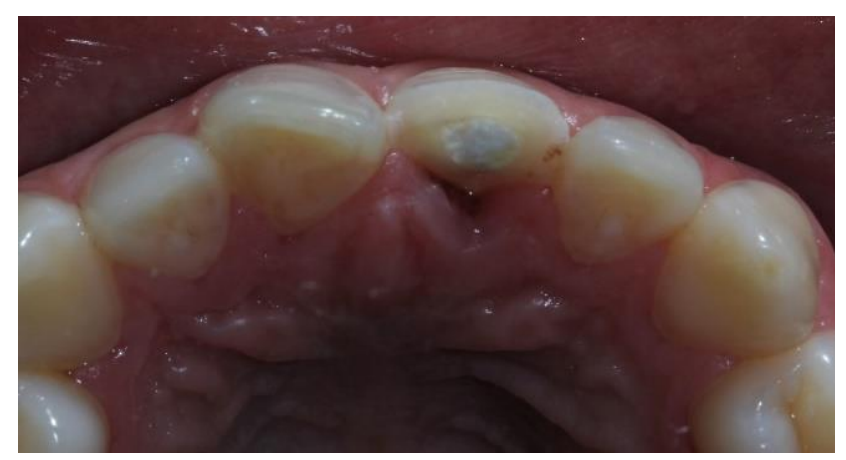

Fig. 3. Tratamiento de Conducto del Incisivo Central Superior.

\section{Preparación del Fragmento Dentario y Diente rema- nente}

A partir de la longitud de trabajo obtenida de la endodoncia en la que se toma como referencia la parte vestibular obteniendo una medida $18 \mathrm{~mm}$ se procede a desobturar el conducto respetando como dicta la literatura al menos entre 3 a $5 \mathrm{~mm}$ de sellado endodóntico a nivel apical, lo cual asegura la hermeticidad apical. ${ }^{7}$

Se da inicio con la desobturación del conducto utilizando fresas Gates Glidden 1 y 2 (Dentsply, Maillefer), ya que por su punta roma aseguran el riesgo de perforación y una menor fricción, generando menor calor durante su utilización, a continuación, con la broca 0.5 y 1 de FGM (Brasil), este poste fue seleccionado de acuerdo a la anatomía y grosor del espacio del conducto observado en la radiografía.

Se procede a colocar el poste prefabricado con la técnica de grabado total, adhesivos y resinas compuestas.

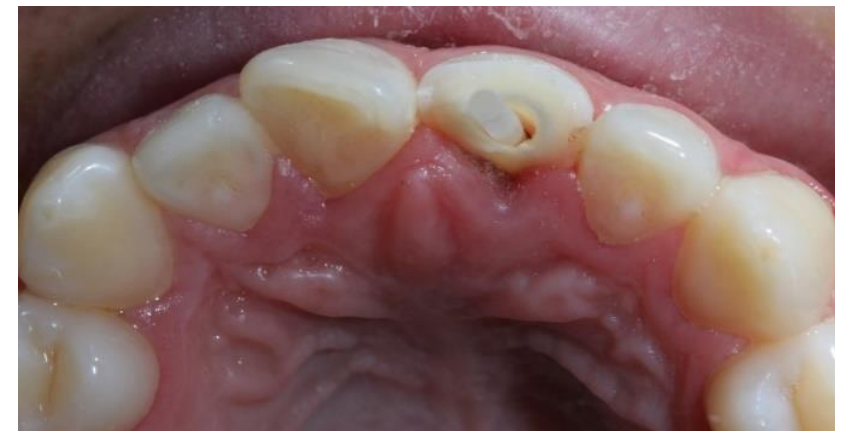

Fig. 4. Desobturación del conducto y prueba del poste

Antes de la cementación se prueba el poste para su ajuste, y proceder a su corte en caso de ser necesario para que no exista interferencia con el fragmento dentario.

\section{Cementación del poste}

El poste debe ser limpiado con alcohol y evaporado con aire a presión libre de contaminación; el cemento a utilizarse es el Paracore Cement (Coltene).

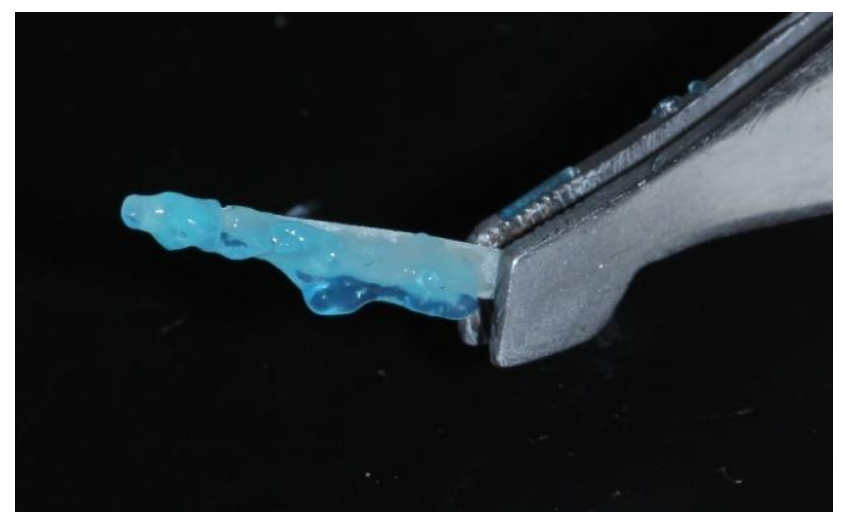

Fig. 5. Colocación de ácido ortofosfórico en el poste

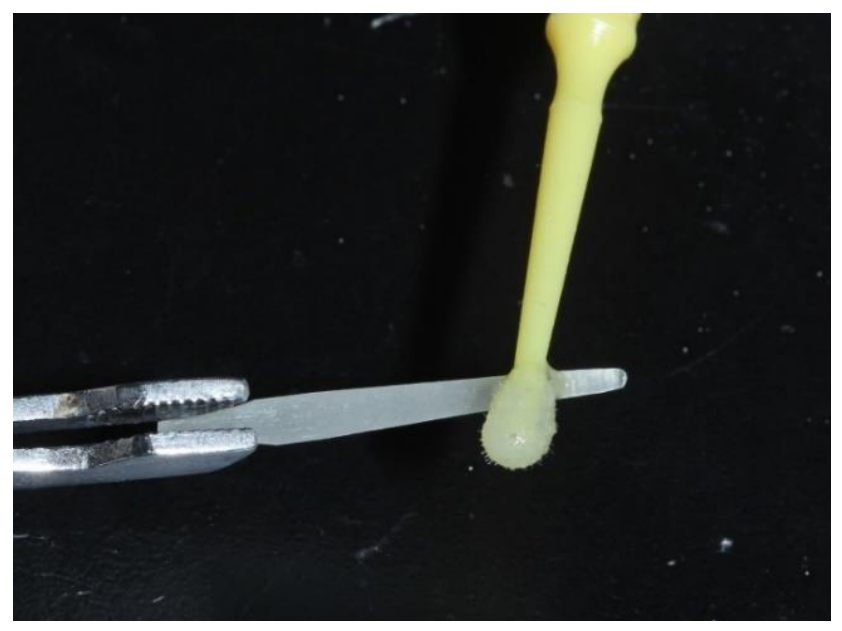

Fig. 6. Colocación de adhesivo en el poste

Para la preparación del conducto se coloca ácido autograbante por 20 segundos (Coltene) se retiran los excesos con puntas de papel estéril, luego se aplica el sistema adhesivo autograbante (Coltene) de acuerdo a las indicaciones del fabricante, se retiran los excesos con puntas de papel estéril, y se fotocura por 20 segundos. Sobre una punta dispensadora se coloca la extensión (elongation tip) y se lleva el cemento (Paracore Cement Coltene) desde el fondo del conducto hacia la superficie para asegurar el llenado total del mismo y evitar burbujas de aire dentro de la mezcla.

Al colocar el poste no se debe exceder la cantidad de cemento ya que puede afectar el asentamiento del fragmento coronario. Por ese motivo se debe realizar una fotopolimerización inicial por 2 segundos para remover fácilmente los excesos de cemento, posteriormente se procede a polimerizar 20 segundos por todas las caras de la pieza, 
como se recomienda en los protocolos de la cementación adhesiva de cualquier restauración, también se debe esperar 5 minutos después de la foto polimerización respetando las indicaciones del fabricante.

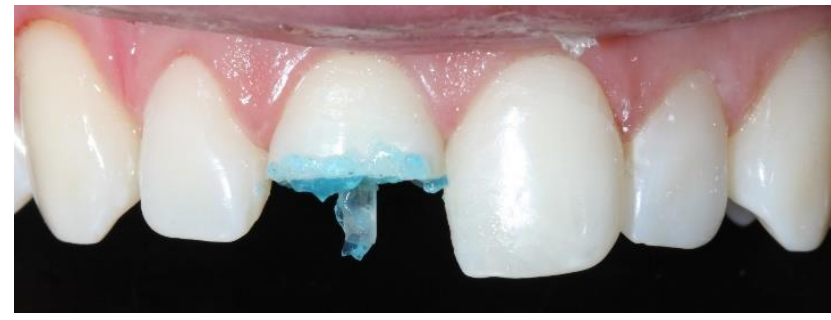

Fig. 7. Grabado acido

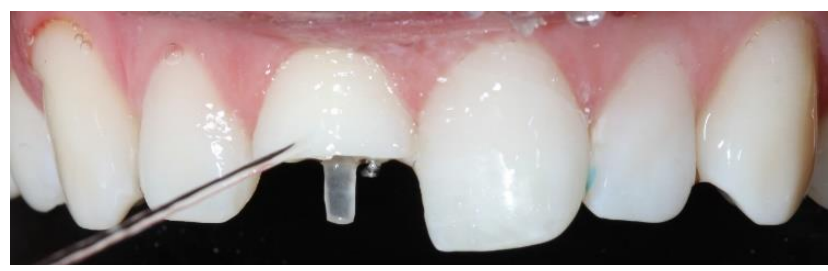

Fig. 8. Lavado de las superficies

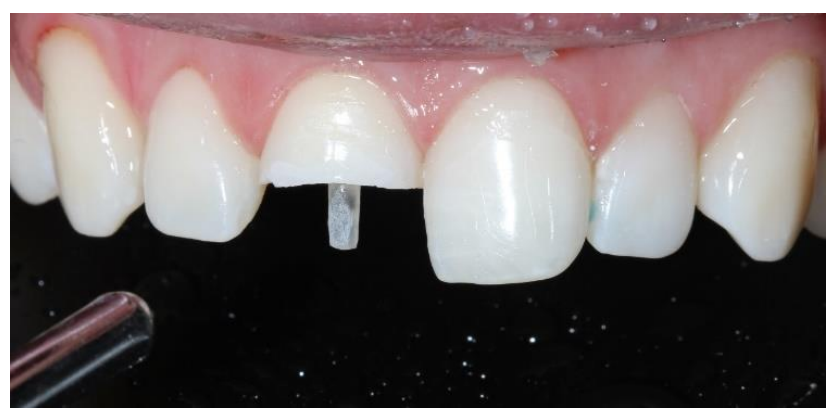

Fig. 9. Secado de superficies

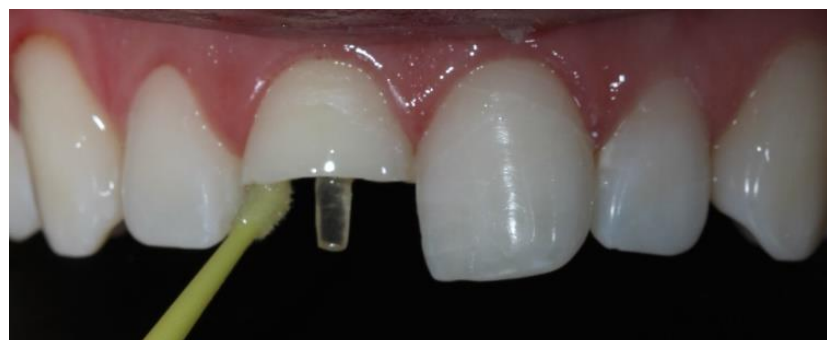

Fig. 10. Colocación de adhesivo

\section{Colocación y adhesión del fragmento}

Se prueba el fragmento coronal y se verifica que exista una buena adaptación y en caso de no existir es necesario modificarlo mediante una preparación con la pieza de mano de alta velocidad para conseguir el asentamiento adecuado, también se realiza un biselado para mejorar la adhesión y la estética.

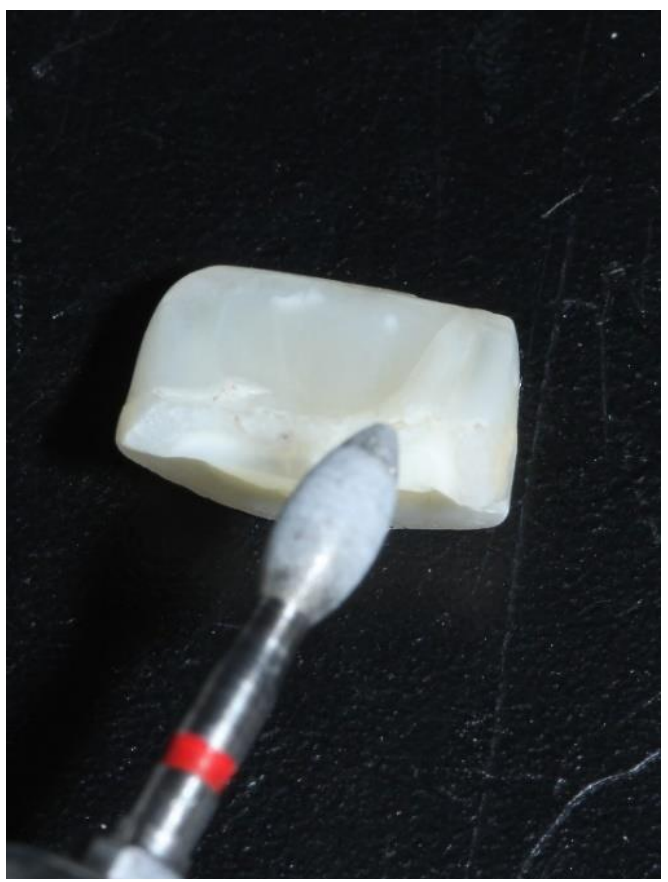

Fig. 11. Biselado del Fragmento

Luego de ello se procede a realizar el grabado ácido por 20 segundos con ácido fosfórico al $37 \%$, se lava el doble de tiempo, para eliminar cualquier residuo, se seca con chorro de aire a $1 \mathrm{~cm}$ de distancia por 5 segundos, se aplica el adhesivo (single bond 3M) con difusión activa, lo cual mejora la penetración del adhesivo, se seca con jeringa de aire para ayudar a la evaporación del solvente. A continuación, se lleva la resina compuesta al interior del fragmento y sobre el remanente dentario; se utilizó hacia vestibular un color A1 Z350 (3M), hacia palatino una capa de opaquer $\mathrm{O} 3(3 \mathrm{M})$ y en la parte externa un esmalte E1 $(3 \mathrm{M})$, para conseguir un resultado estético satisfactorio.

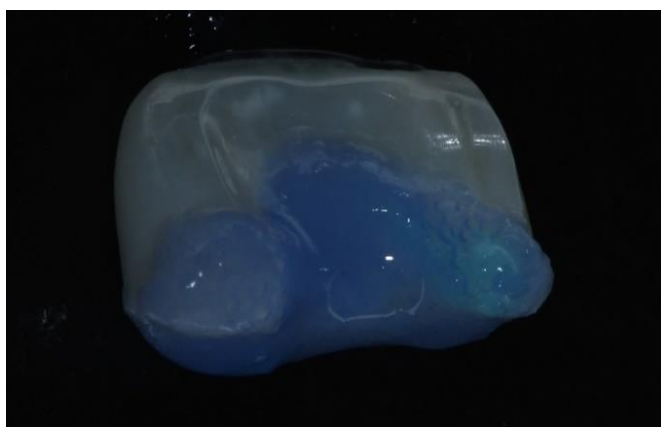

Fig. 12. Grabado ácido del fragmento 


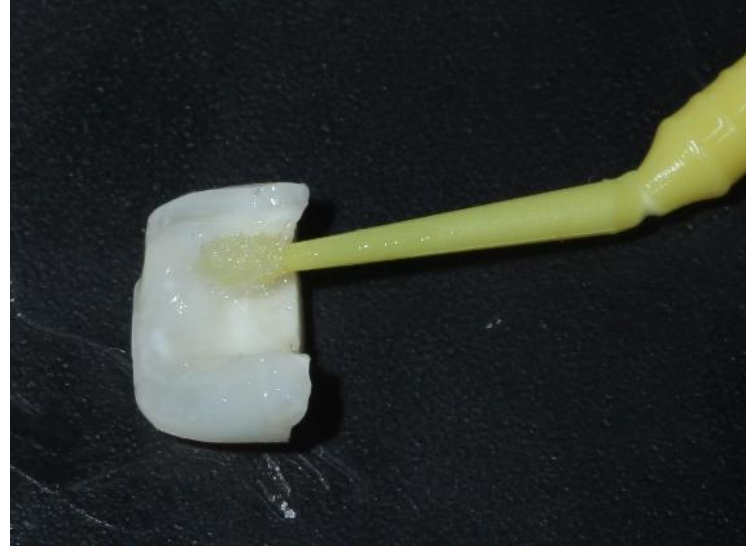

Fig. 13. Colocación del sistema adhesivo

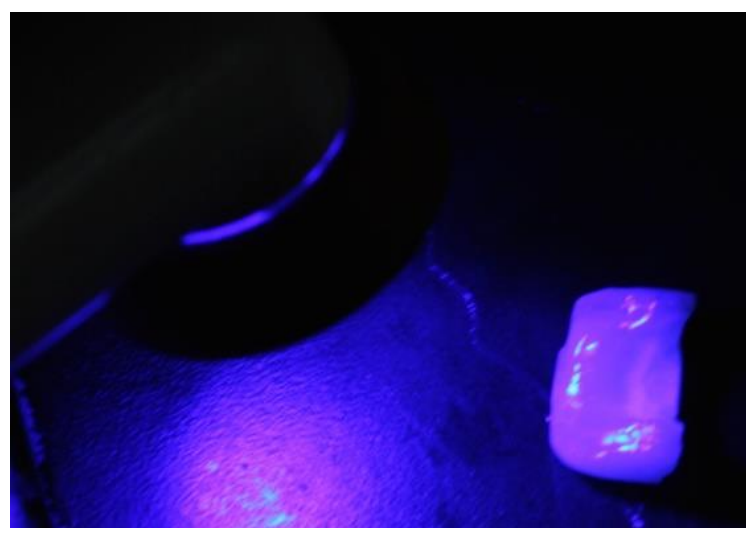

Fig. 14. Colocación del fragmento

Se retiran todos los excesos y se procedió a fotocurar con una lámpara LED radii plus (SDI), después de cada incremento de resina, y una fotopolimerización final colocando glicerina para eliminar la capa inhibida por oxígeno, evitando el cambio de color ya que esta capa es propensa y sensible a ello.

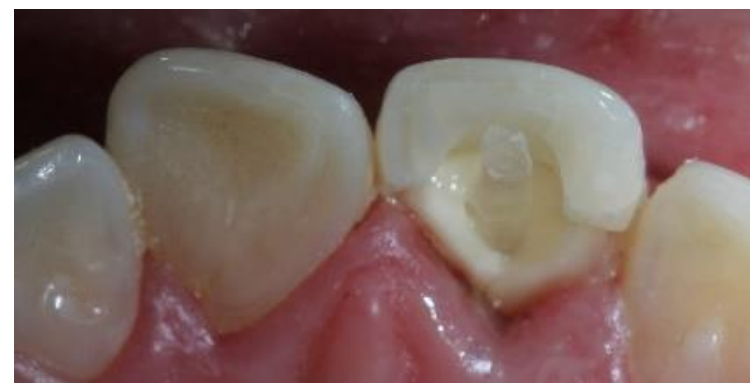

Fig. 15. Colocación del fragmento

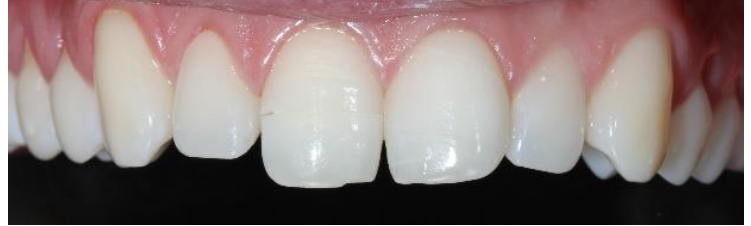

Fig. 16. Colocación del fragmento

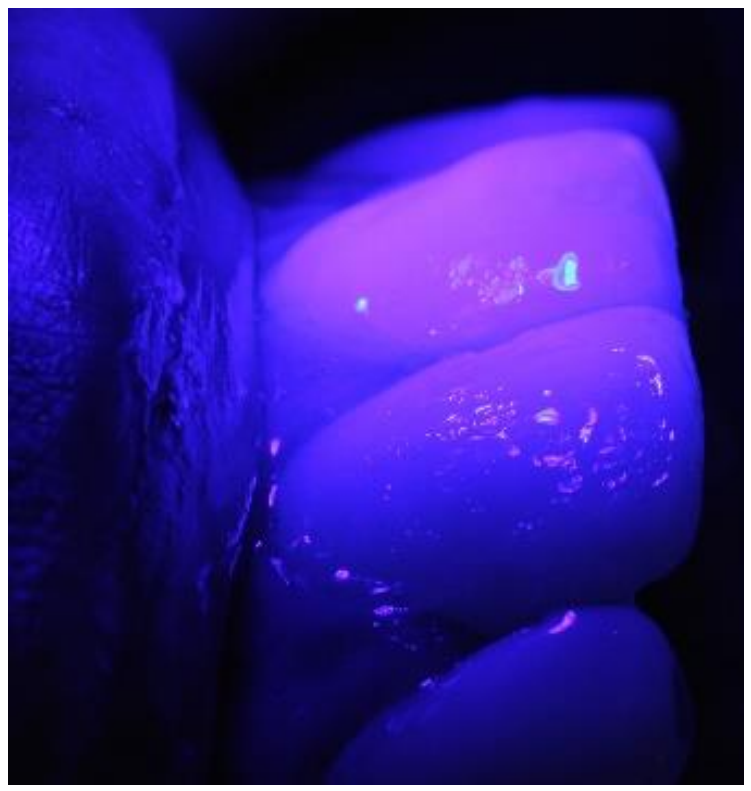

Fig. 17. Aplicación de gel de glicerina y fotocurado

Se realiza el ajuste oclusal utilizando papel de articular (AccuFilM II, Parkell), posteriormente se realiza el desgaste con fresas de grano medio (0,40 diámetro Kommet), luego realizamos el pulido con discos soflex de (3M), discos de lija, y finalmente con un disco de fieltro y pasta diamantada (TDV) realizamos el pulido final.

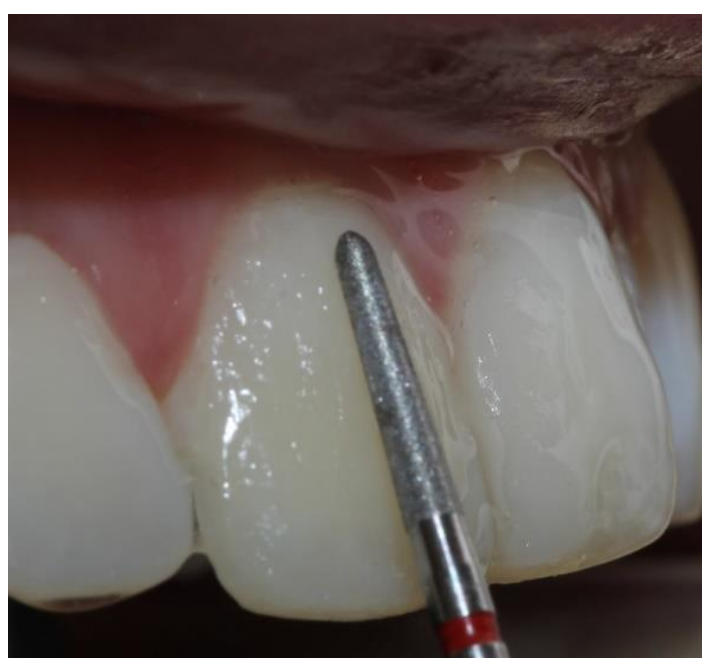

Fig. 18. Pulido fresa grano Medio 


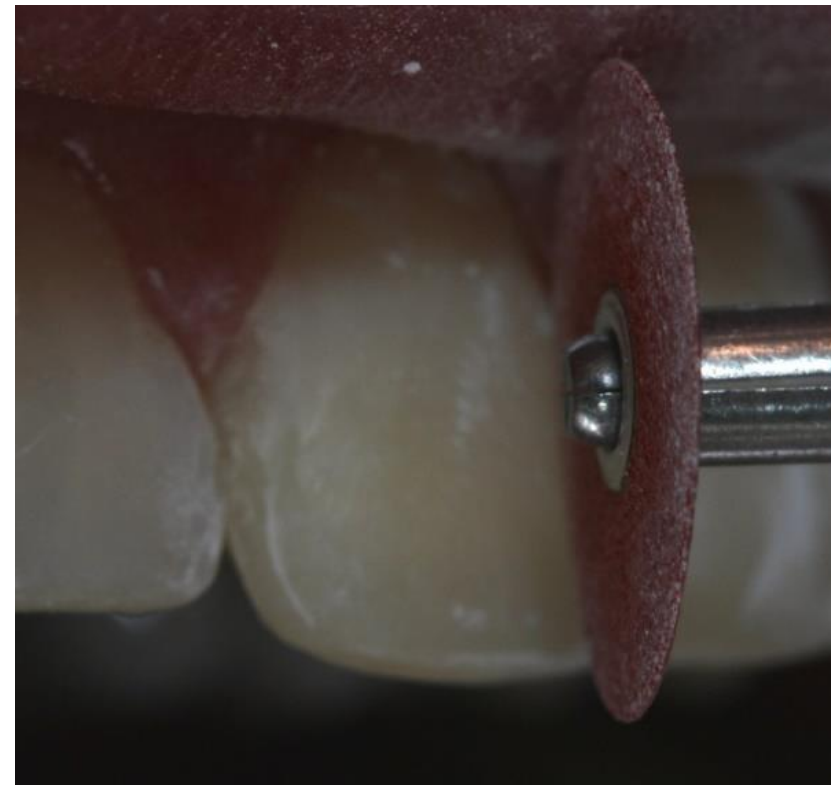

Fig. 19. Pulido discos soflex

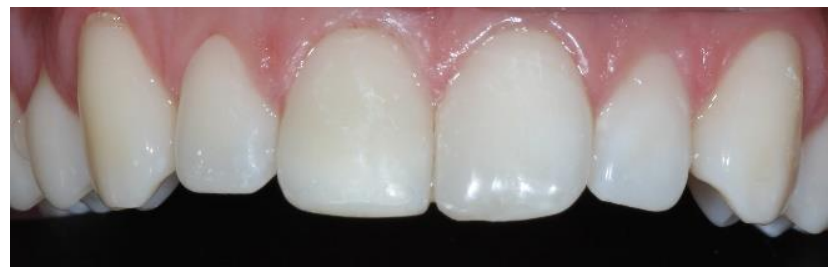

Fig. 20. Restauración Final

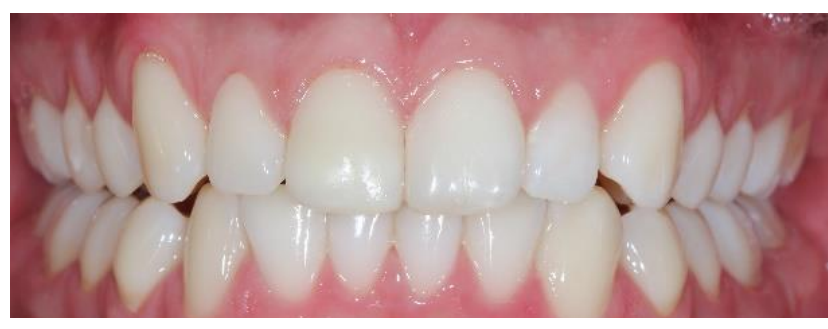

Fig. 21. Final en oclusión

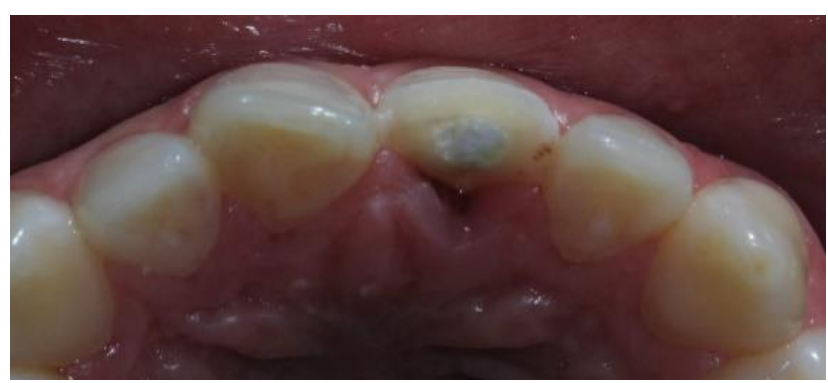

Fig. 22. Vista vestibular antes

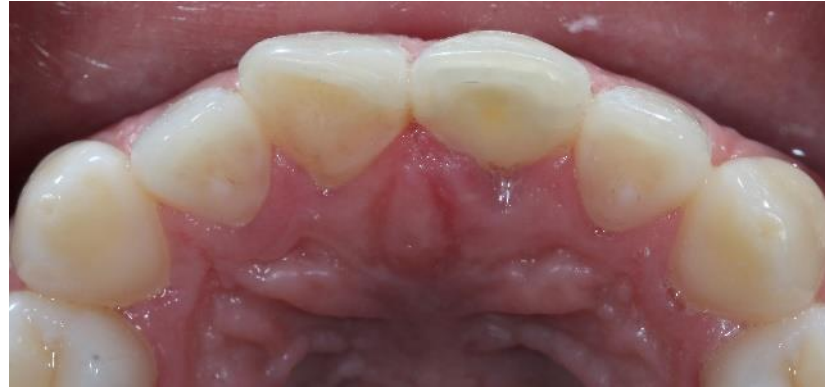

Fig. 23. Vista vestibular después

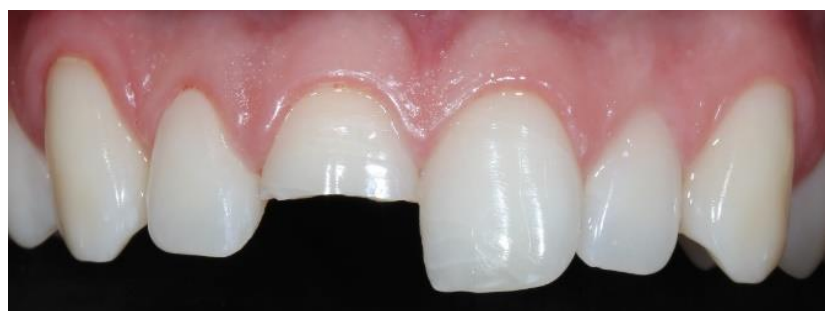

Fig. 24. Fractura Antes

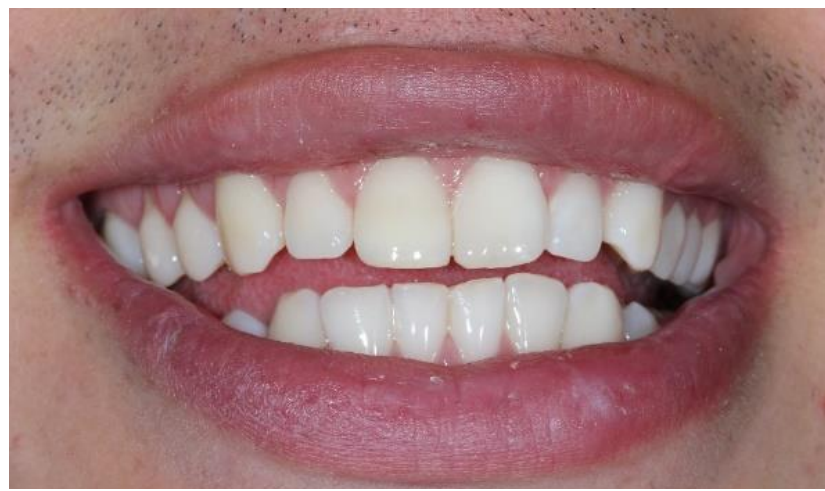

Fig. 25. Restauración y sonrisa final

\section{Discusión}

Las lesiones coronarias traumáticas se establecen dentro de las más concurridas en la dentición permanente ${ }^{2,8,9}$

La causa más común de estos accidentes lo forman los estilos de vida del paciente, junto con la propensión de asumir riesgos a través de ignorar las formas de prevención establecidas para impedir estos traumatismos. Del mismo modo, se nombran como elementos de alto riesgo, las caídas accidentales, los deportes de riesgo (en especial entre los jóvenes), incidentes de tránsito ${ }^{10,11}$ riñas y deportes en bicicleta $^{9}$

Hay variadas opiniones para el tratamiento de las fracturas coronarias. A pesar de estas variaciones es importante alcanzar un diagnóstico individual, donde estén constatados la edad, el alcance de la fractura de la corona, la posible existencia del compromiso pulpar, el nivel de desarrollo 
radicular, el estado de las estructuras adyacentes, el periodo que ha pasado desde el traumatismo hasta el momento de la observación, la constatación de sintomatología, la manifestación a otras pruebas de vitalidad y, la problemática funcio-estética-psicológica.

La medida terapéutica que se debe tomar en primer lugar para estas situaciones, es el rediseño de la pieza o de parte de ella, cuando se ha conservado,,${ }^{9,12}$ dado que es lo más funcional, lo más estético y conservacionista, ya que posibilita restablecer la traslucidez, la opalescencia, la fluorescencia, las formas de la pieza original, junto a su textura. ${ }^{9,13}$ Otra ventaja es que hay un operatorio más reducido, ${ }^{9}$ y la frecuencia de desgaste y abrasión del diente es la misma que para una pieza dentaria intacta ${ }^{13}$

En la actualidad, la técnica de acondicionamiento de la dentina, se reconoce como una alternativa terapéutica muy viable en la reposición del fragmento en las situaciones coronales que no revistan complejidad, estableciendo la necesidad de un fragmento de esmalte para su reanclaje (dentina intacta). ${ }^{2} \mathrm{Al}$ adherir el fragmento, es posible hacer una postpreparación junto a la línea de fractura con resina compuesta, mejorando estética y la retención ${ }^{8}$

La unión de un fragmento se constató por primera vez a mediados del siglo pasado (1964), gracias a un odontopediatra de la universidad de Odontología de Hadassah (Israel). También se debe tener en cuenta, de que fue el doctor Simonsen el pionero en protocolizar la forma de preparación para conseguir acoplar la parte fracturada al diente. Esta técnica se fundamentaba en conseguir un bisel de cuarenta y cinco grados sobre el esmalte fragmentado y en la pieza dental; previamente con un grabado ácido y utilización de resina ${ }^{8}$

Es muy reconocida esta técnica de grabado ácido, preparación de la dentina y posteriormente la utilización de la resina compuesta para la reinserción de partes o fragmentos en las lesiones coronarias. Se ha comprobado, que la eficacia de unión y el nivel de retención de estos fragmentos son equivalentes a la superficie de esmalte utilizable para la aplicación del grabado ácido ${ }^{11}$

Por otro lado, las investigaciones alcanzadas proponen otras técnicas de reinserción, produciendo una divergencia de opiniones sobre las más convenientes. Técnicas investigadas con la utilización de biseles, chaflanes, ranuras para la retención en esmaltes y en dentinas, y en sobre contorneado a través de resina compuesta. ${ }^{9}$

En la actualidad hay un debate abierto sobre esta temática, dado que no hay consenso en los diferentes investigadores. Algunos profesionales explican que aplicar preparaciones dentarias extras para exponenciar la retención del fragmento, como pueden ser biseles, chaflanes y ranuras de retención en esmaltes y dentinas, provocan una perturbación en la pieza lacerada.

Actualmente, no existen referencias investigativas que sustenten esta técnica, por lo que está usualmente descartada por varios motivos como enumera Ghandi, K. et al. (2006): en primer término, sino es preparada la superficie adyacente a la lesión, el grabado ácido no será tan efectivo; en segundo término, esta metodología constantemente deja una superficie de sobre contorno que desfavorece el aspecto estético; en tercer término, los espacios de preparación permanecen débiles y consecuentemente se distorsionan o se vuelven a traumatizar con frecuencia, acrecentando de esta manera las posibilidades de fracaso. Otros argumentos que se exponen para la preparación final del esmalte son las siguientes: ampliación de la fuerza de unión para el esmalte y la resina compuesta, dado que se dispone de una mayor superficie de esmalte aplicable para la unión; se disminuye la contracción por polimerización; la policromía que resulta de la técnica es uniforme y favorece la imagen estética; y, por último, está técnica es más resistente en el tiempo ${ }^{11}$

En los casos en que la reposición del fragmento no es viable, es recomendable la utilización de resina compuesta para la reconstrucción de la pieza fracturada, ${ }^{8,9,13}$ con una técnica operatoria adecuada para lograr una restauración estética y perdurable.

\section{Conclusiones}

Son habituales los daños traumáticos en la dentición permanente, con bastante asiduidad en las lesiones coronarias, por lo que es importante su conocimiento entre los profesionales de la odontología, para que se pueda ofrecer un servicio adecuado a las personas que acuden a las consultas con la urgencia.

Un diagnóstico preciso del caso, concluido por una pormenorizada anamnesis, una observación clínica y radiográfica de la zona en cuestión, será necesario para construir un plan de tratamiento idóneo.

Para este tipo de tratamiento los fragmentos se deben conservar, los mismos que deben mantener su hidratación y con la técnica de collage se devolverá la morfología y anatomía de las piezas dentarias devolviendo al paciente su funcionalidad y estética.

El pronóstico que resulte, será definido por la extensión de la fractura, por la aparición de otras lesiones conexas en los tejidos de soporte, y por la forma de tratamiento que se aplique.

Entre las diferentes técnicas de tratamiento que se reconocen, la reinserción del fragmento fracturado o collage es la elección más clásica y en la práctica, la más invariable. A pesar de esto, se exhorta a no usarla de manera única, sino simultáneamente con preparaciones extras para propiciar su resistencia y estéticamente correcta con refuerzos de resina, chaflanes, biseles y retenciones.

Se considera difícil garantizar la longevidad de la parte fracturada al diente, por lo que es básico contar con el apoyo, cuidado del paciente y una técnica adecuada por parte del profesional lo que hará que se marque la diferencia disminuyendo el porcentaje de tratamientos más invasivos a corto o largo plazo.

\section{Referencias Bibliográficas}

1. Dietschi D, Jacoby T, Dietschi JM, Schatz JP. Treatment of traumatic injuries in the front teeth: restorative aspects 
in crown fractures. Pract Periodontics Aesthet Dent. 2000 Oct;12(8):751-758. Available from: https://www.ncbi. nlm.nih.gov/pubmed/11404871.

2. Andreasen JO, Ravn JJ. Epidemiology of traumatic dental injuries to primary and permanent teeth in a Danish population sample. International Journal of Oral Surgery. 1 1972;1(5):235-239.

3. García FJM, Fernández RE. ADHESION DE FRAGMENTOS DENTALES; 2013. accessed 27. Dec. 2017]. Available from: http://www.rodyb.com/wp-content/uploads/2013/08/ ADHESION-DE-FRAGMENTOS-13.pdf.

4. Bouza Vera M, Gou Godoy A, Barrios Rodríguez T. 17. SK M, FAP R, LF C, AY F, RFL M, J M. Efecto del Alternativa de tratamiento de fractura no complicada de corona por medio de adhesión del fragmento. Presentación de un caso. Revista Médica Electrónica. 2014;36:763770 .

5. Rosero Alvario LE. Técnica unión - collage de frag- 18 mento dental con matriz de acrílico en el tratamiento de incisivos superior con fractura del tercio incisal. Repositorio Institucional de la Universidad de Guayaquil. 2012 Jun;Available from: http://repositorio.ug.edu. ec/handle/redug/2947.

6. Zambrano GA, Rondón RG, Sogbe R. Fracturas Coronarias de Dientes permanentes y alternativas de tratamiento - Revisión de la Literatura. Revista Latinoamericana de Ortodoncia y Odontopediatría. 2012;p. 1-10. Available from: https://www.ortodoncia.ws/publicaciones/2012/art$34 /$.

7. Abramovitz L, Lev R, Fuss Z, Metzger Z. The unpredictability of seal after post space preparation: a fluid transport study. Journal of endodontics. 2001;27:292-295.

8. García Ballesta C, Mendoza A. Traumatología Oral en Odontopediatría. Diagnóstico y Tratamiento Integral. vol. 1. Madrid, España: Ergon- Editorial Médica; 2003.

9. Díaz JA. Fracturas coronarias en incisivos centrales maxilares; seguimiento de 2 años y resultado clínico en niños. Revista International Journal of Odontostomatology. 2008;2:83-94.

10. Viduskalne I, Care R. Analysis of the crown fractures and factors affecting pulp survival due to dental trauma. Stomatologija, Baltic Dental and Maxillofacial Journal. 2010;12:109-115.

11. Ghandi K, Nandlal B. Effect of enamel preparations on fracture resistance of composite resin buildup of fractures involving dentine in anterior bovine teeth: An in vitro study. Journal of Indian Society of Pedodontics and Preventive Dentistry. 2006;24:69-75. Available from: http://www.jisppd.com/text.asp?2006/24/2/69/26019.

12. Butler T, Lazo S, Lazo G, al e. Éxitos y fracasos de fracturas dentarias con técnica de collage. Revista Odontológica de México. 2010;IV:1-8. Available from: http://www. intramed.net/contenidover.asp?contenidoID $=71436$.

13. Goenka P, Marwah N, Dutta S. Biological approach for management of anterior tooth trauma: Triple case report. Journal of Indian Society of Pedodontics and Preventive
Dentistry. 2010;28:161-164. Available from: http://www. jisppd.com/text.asp?2010/28/3/223/73791.

14. Berman LH, Kuttler S. Fracture necrosis: diagnosis, prognosis assessment, and treatment recommendations. J Endod. 2010 Mar;36(3):442-446.

5. 15 Andreasen JO, Andreasen FM, Andersson L. Textbook and Colors Atlas of Traumatic Injuries to the Teeth. Copenhagen: Blackwell Publishing Ltd; 2007.

6. Éxitos y fracasos de fracturas dentarias con técnica de collage; 2011. [Online; accessed 27. Dec. 2017]. Available from: http://www.intramed.net/contenidover. asp? contenidoID=71436. biselado en la infiltración marginal de restauraciones clase IV, en resina compuesta; 2015. [Online; accessed 27. Dec. 2017]. Available from: http://www.rodyb.com/wpcontent/uploads/2014/12/vol-4-N1-bisel1.pdf.

. Baratieri LN, Martínez Santos Fernandes A. Estética: restauraciones adhesivas directas en dientes anteriores fracturados. Colombia: Santos. AMOLGA; 2004. OCLC: 70160735 .
Recibido: 24 de octubre de 2017

Aceptado: 11 de diciembe de 2017 